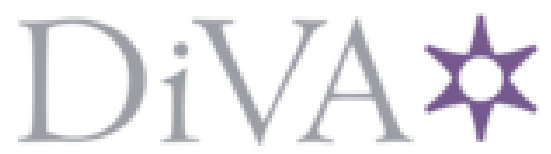

http://www.diva-portal.org

\title{
Postprint
}

This is the accepted version of a paper presented at IEEE 15th International Conference on Environment and Electrical Engineering (EEEIC), JUN 10-13, 2015, Rome, ITALY.

Citation for the original published paper:

Yuan, Z., Hesamzadeh, M R. (2015)

A Hierarchical Dispatch Structure for Distribution Network Pricing.

In: 2015 IEEE 15TH INTERNATIONAL CONFERENCE ON ENVIRONMENT AND

ELECTRICAL ENGINEERING (IEEE EEEIC 2015) (pp. 1631-1636). IEEE

N.B. When citing this work, cite the original published paper.

"C 2016 IEEE. Personal use of this material is permitted. Permission from IEEE must be obtained for all other uses, in any current or future media, including reprinting/republishing this material for advertising or promotional purposes, creating new collective works, for resale or redistribution to servers or lists, or reuse of any copyrighted component of this work in other works."

Permanent link to this version:

http://urn.kb.se/resolve?urn=urn:nbn:se:kth:diva- 180378 


\section{A Hierarchical Dispatch Structure for Distribution Network Pricing}

\author{
Zhao Yuan \\ Electricity Market Research Group \\ KTH Royal Institute of Technology \\ Stockholm, Sweden \\ Email: yuanzhao@kth.se
}

\author{
Mohammad Reza Hesamzadeh \\ Electricity Market Research Group \\ KTH Royal Institute of Technology \\ Stockholm, Sweden
}

\begin{abstract}
This paper presents a hierarchical dispatch structure for efficient distribution network pricing. The dispatch coordination problem in the context of hierarchical network operators are addressed. We formulate decentralized generation dispatch into a bilevel optimization problem in which main network operator and the connected distribution network operator optimize their costs in two levels. By using Karush-Kuhn-Tucker conditions and Fortuny-Amat McCarl linearization, the bilevel optimization problem is reduced into a mixed-integer linear programming (MILP) problem. Equivalence between proposed hierarchical dispatch and centralized dispatch is proved. The model is solved in GAMS platform. IEEE 14-bus meshed network and IEEE 13-node radial network are connected to be an illustrative example offering numerical dispatch results. Three scenarios representing distributed generation (DGs) successive development stages are analyzed. Hierarchical dispatch achieves same results as traditional centralized dispatch in the three considered scenarios. Distribution network nodal prices are obtained. Intrinsic advantages of the proposed hierarchical dispatch are to reduce the dispatch complexity with increasing DGs penetration and provide distribution locational marginal prices (DLMP).
\end{abstract}

Index Terms-Hierarchical dispatch, distribution network pricing, distributed generation.

\section{NOMENCLATURE}

Indices:

$i$ Index for generation units from $i_{1}$ to $i_{I}$.

$j$ Index for demand units from $j_{1}$ to $j_{J}$.

$n$ Index for buses from $n_{1}$ to $n_{N}$.

$l \quad$ Index for branches from $l_{1}$ to $l_{L}$.

$k$ Index for networks from $k_{1}$ to $k_{K}$.

Constants:

$c_{i} \quad$ Variable cost of generation unit $i$.

$G_{i} \quad$ Generation capacity cost of generation unit $i$.

$D_{j} \quad$ Demand power of demand unit $j$.

$B_{l} \quad$ Branch susceptance $l$.

$F_{l} \quad$ Power transmission capacity of branch $l$.

$A_{k} \quad$ Incidence matrix of network $k$.

$N_{k} \quad$ Node to generation incidence matrix of network $k$.

Variables:

$g_{i} \quad$ Dispatched power for generation unit $i$.

$f_{l} \quad$ Power flow for branch $l$.

$\delta_{n} \quad$ Voltage phase angle for bus $n$.

$C_{k} \quad$ Generation cost of network $k$.
Dual Variables(with corresponding constraints):

$u_{i}^{\min }$ Lower production bound of generation unit $i$.

$u_{i}^{\max }$ Upper production bound of generation unit $i$.

$u_{n}^{\min } \quad$ Minimum voltage angle of bus $n$.

$u_{n}^{\max } \quad$ Maximum voltage angle of bus $n$.

$u_{l}^{\min }$ Power transmission lower bound of branch $l$.

$u_{l}^{\max }$ Power transmission upper bound of branch $l$.

$\lambda_{n}^{p} \quad$ Power flow balance of bus $n$.

$\lambda_{l}^{p} \quad$ Power flow of branch $l$.

\section{INTRODUCTION}

Electricity industry deregulation around the world is reshaping power system operation and management from a vertical top-down manner to be more bidirectional and distributed. Coupled with smart grid development, efficient operation of power system is of major importance, [1]. However, distribution grids with increasing penetration of distributed generators (DGs), smart meters, demand response and electric vehicles (EVs) remain lacking proper locational marginal price signals for the growing prosumers as well as third-party innovation, [2]. A power grid always with over-invested distribution network to eliminate any possible occur of congestion can not be called smart at least in a economic point of view. Benefits of distribution pricing have been concluded as rewarding DGs for reducing losses [3], electric vehicle (EV) charging management [4] and boosting demand side responses [5], [6]. Recently proposed Distributed System Platform Provider (DSPP) concept by New York Public Service Commission redefines distribution utility as platform and serves as an interface for various services, products and market players, [7]. Such a vision highlights pivotal role of distribution utility. Demand side responses behind the meter will challenge the new distribution system operator (DSO) in the future, [8]. In [9], progress of long-run incremental cost pricing (LRIC) and forward cost pricing (FCP) as two emerged economic charging methodologies for Great Britain distribution pricing reform are introduced. Though these methodologies offer locational messages the complexity hinders industry to implement LRIC and FCP in high voltage and low voltage (HV/LV) network. Considering large number of nodes in distribution networks, complexity involved in distribution pricing should be addressed properly. In this sense, decentralized dispatch is 
attractive to smart grid, [10]-[12]. To address the complexity, decentralized optimal power flow (OPF) is calculated by Lagrangian Relaxation decomposition procedure, [13]. Reference [14] reduces high voltage (HV) radial distribution networks to simple networks by feeder reduction techniques. In [15], economic dispatch is obtained in distributed average consensus problems by self-organizing dynamic agents. [16] presents a distributed multi-agent paradigm to calculate DLMP. Recently, [4] uses DC power flow approach in distribution system to determine DLMP. Although past research confirms possibility of distributed dispatch in smart grid, dispatch coordination between hierarchical operators with limited network information sharing is rarely discussed. This issue is very important for the operation of a future pan-European electricity market.

In this paper, a hierarchical dispatch structure is proposed and explored for dispatch coordination in the context of hierarchical network operators. The main aim is to provide practical and efficient distribution network pricing approach. The mathematical equivalence between our proposed hierarchical dispatch and centralized dispatch is proved and demonstrated. Distribution network nodal prices are obtained. The rest of this paper is organized as follows. Section II presents a bilevel optimization model for hierarchical dispatch and the equivalent linearization. Section III provides a illustrative dispatch task example, numerical results for distribution network pricing. Section IV concludes advantages of the proposed hierarchical dispatch structure.

\section{Hierarchical Dispatch In SMART GRID}

\section{A. Hierarchical Dispatch Structure}

Suppose $\boldsymbol{x}$ and $\boldsymbol{y}$ are decision variables of main network and sub-network operators (one can assume the main network operators here to be TSOs and sub-network operator to be DSOs but we prefer to keep these terminologies of 'main network operator' and 'sub-network operator' only in the purpose of being suitable for more general cases). Assuming linear objective functions and constraints (nonlinear cases are quite similar). The whole economic dispatch problem of main network and sub-network can be written as:

$$
\begin{array}{ll}
\text { Minimize } & c x+d y \\
\text { subject to } & A x+B y \leq b \\
& x \geq 0, y \geq 0
\end{array}
$$

Assuming decomposition conditions hold for this problem (which is valid in many real cases), this minimization problem can be rewritten as,

$$
\begin{aligned}
& \text { Minimize } c x+\alpha \\
& \text { subject to } x \geq 0
\end{aligned}
$$

Where,

$$
\begin{aligned}
\alpha(x)=\text { Minimize } & d y \\
\text { subject to } & B y \leq b-A x \\
& y \geq 0
\end{aligned}
$$

This mathematical derivation inspires a hierarchical dispatch scheme. The main network operator minimizes its own cost taking into account the $\alpha(\mathbf{x})$ cost of sub-network and main network constraints. The sub-network operator minimizes its own operation cost (6) subject to its local constraints (7)-(8) given the decision of main network operator. This formulation is derived as a bilevel optimization problem in part B of this section model equations (9)-(20).

The proposed hierarchical dispatch structure is depicted in Figure 1. Main network could be regional transmission network and sub-networks are the corresponding connected distribution networks. Conventional generators (thermal, nuclear and gas generators) and dispatchable DGs are dispatched to meet demand by dispatch targets after bidding. Main network operator only dispatches the generation assets located in his own responsible region. Dispatch task of subnetwork is taken over by individual regional sub-network operator or distribution network operator, which reduces complexity of large-scale power-system dispatch. The main network operator as the leader takes the first move considering all generation costs. Sub-network operators as the followers consider only local costs. The powers transported between main network and sub-networks are the only physical information about the sub-network that main network operator holds in hands.

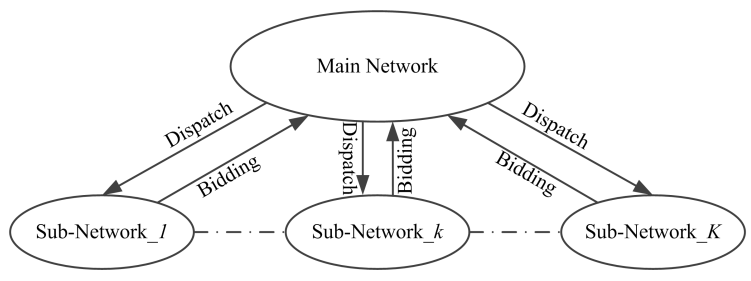

Fig. 1. Hierarchical Dispatch Structure

It is worth to note that achieving economic operation of main network and all subnetworks is still the final goal of hierarchical dispatch. As long as sub-network or distribution network operators communicate correct cost functions as bidding functions to the main network operator, hierarchical dispatch should, in principle, reach results same as centralized dispatch. We will show later in section III that the bidding functions from sub-networks demonstrate reverse merit-order characteristics. Hierarchical dispatch is a trade-off between dispatch complexity and efficiency. This can also be preferable as cyber security of smart grid significantly arises. If one cyber attack seriously happens to a traditional centralized dispatch centre, all components in the whole system are possible to be impacted. The worst consequence is overall shutdown in the operation area. Whereas in hierarchical dispatch, single attack in one dispatch centre can not directly affect assets located in other areas. Cybersecurity of the system is enhanced.

\section{B. Bilevel Optimization Model}

In hierarchical dispatch, main network operator minimizes generation cost of the whole area including transmission network and the connected distribution networks. Distribution 
network operators are responsible to minimize generation cost of local networks considering power flow from other networks. The bilevel optimization model proposed here is flexible to extend to cases where multiple operators with distribution networks connected to a single transmission network are considered. All distribution network operators' minimization problems are in the second level. Though $\mathrm{R} / \mathrm{X}$ ratio of branches in distribution system are higher than in transmission system, we neglect distribution losses and use DC power flow calculations for simplicity (similar with reference [4]). This DC power flow approach could result in distorted dispatch targets and will be addressed in future works. The main goal here is to design a feasible dispatch scheme to facilitate efficient distribution pricing and gain useful insights from a simple model. Only stringently-controllable DGs are considered in the model.

Model:

$$
\underset{g_{i}, \forall i \in k_{1}}{\operatorname{Minimize}} \quad C=\sum_{k} C_{k}
$$

subject to

$$
\begin{aligned}
& \sum_{i \in n} g_{i}-\sum_{j \in n} D_{j}=A_{k_{1}}^{T} f_{l}, \forall n, l \in k_{1} \cup k_{1 \_2} \\
& f_{l}=B_{l} A_{k_{1}} \delta_{n}, \forall n, l \in k_{1} \cup k_{1 \_2} \\
& 0 \leq g_{i} \leq G_{i}, \forall i \in k_{1} \\
& 0 \leq f_{l} \leq F_{l}, \forall l \in k_{1} \cup k_{1 \_2} \\
& -\pi \leq \delta_{n} \leq \pi, \forall n \in k_{1} \\
& \underset{g_{i}, \forall i \in k_{2}}{\text { Minimize } C_{k_{2}}} \\
& \text { subject to } \\
& \quad \sum_{i \in n} g_{i}-\sum_{j \in n} D_{j}=A_{k_{2}}^{T} f_{l}: \lambda_{n}^{p}, \forall n, l \in k_{2} \cup k_{1 \_2} \\
& \quad f_{l}=B_{l} A_{k_{2}} \delta_{n}: \lambda_{l}^{p}, \forall n, l \in k_{2} \\
& \quad 0 \leq g_{i} \leq G_{i}: u_{i}^{\text {min }}, u_{i}^{\text {max }}, \forall i \in k_{2} \\
& \quad 0 \leq f_{l} \leq F_{l}: u_{l}^{\text {min }}, u_{l}^{\text {max }}, \forall l \in k_{2} \\
& \quad-\pi \leq \delta_{n} \leq \pi: u_{n}^{\text {min }}, u_{n}^{\text {max }}, \forall n \in k_{2}
\end{aligned}
$$

Where $k_{1 \_2}$ are set of tie-lies between main network and subnetwork. The whole network generation cost minimization problem is stated by (9)-(20). This is an optimization problem (9)-(14) constrained by another optimization problem (15)(20). The main network generation cost minimization problem is stated by (9)-(14) where objective function (9) include generation cost of both main network and sub-network. Only power production from generators located in the main network are the control variables in this upper-lever optimization. Only the main network constraints (10)-(14) are taken into account by the main network operator. Equations (10) represent nodal power flow balance. Equations (11) calculate power flow by DC power flow approach. Constraints (12) are the power production bounds for generation units in the main network. Constraints (13) are the power flow bounds for the transmission lines in the main network. Constraints (14) are the bus voltage angle bounds in the main network. The lowerlevel problem (15)-(20) represents generation cost minimiza- tion for the distribution or sub-network. Control variables in this lower-level optimization are power production from local dispatchable DGs. Equations (16)-(20) are constraints of the distribution network considered by the distribution or subnetwork operator. Equations (16) represent nodal power flow balance. Equations (17) calculate power flow by DC power flow approach. Constraints (18) are the power production bounds for generation units or DGs in the main network. Constraints (19) are the power flow bounds for the distribution lines in the sub-network. Constraints (20) are the bus voltage angle bounds in the sub-network. Equations (16)-(20) are followed by corresponding dual variables with a colon.

\section{Equivalent Linearization}

Assuming cost of power production from all generation units are linear, the lower-level problem (16)-(20) can be replaced equivalently by its KKT conditions. This approach leads to a mathematical programming with equilibrium constraints (MPEC) problem. The KKT conditions of lower-level optimization problem are derived in (21)-(31).

$$
\begin{aligned}
& \sum_{k} \frac{\partial C_{k}}{\partial g_{i}}-u_{i}^{\text {min }}+u_{i}^{\text {max }}+N_{g}^{T} \lambda_{n}^{p}=0 \\
& \lambda_{l}^{p}-A_{k_{2}}^{T} \lambda_{n}^{p}+u_{l}^{\text {max }}-u_{l}^{\text {min }}=0 \\
& -A_{k_{2}}^{T} B_{l} \lambda_{l}^{p}+u_{n}^{\text {max }}-u_{n}^{\text {min }}=0 \\
& \sum_{i \in n} g_{i}-\sum_{j \in n} D_{j}=A_{k_{2}}^{T} f_{l}, \forall n, l \in k_{2} \cup k_{1 \_2} \\
& f_{l}=B_{l} A_{k_{2}} \delta_{n}, \forall n, l \in k_{2} \\
& 0 \leq g_{i} \perp u_{i}^{\text {min }} \geq 0, \forall i \in k_{2} \\
& 0 \leq G_{i}-g_{i} \perp u_{i}^{\text {max }} \geq 0, \forall i \in k_{2} \\
& 0 \leq f_{l}+F_{l} \perp u_{l}^{\text {min }} \geq 0, \forall l \in k_{2} \\
& 0 \leq F_{l}-f_{l} \perp u_{l}^{\text {max }} \geq 0, \forall l \in k_{2} \\
& 0 \leq \delta_{n}+\pi \perp u_{n}^{\text {min }} \geq 0, \forall n \in k_{2} \\
& 0 \leq \pi-\delta_{n} \perp u_{n}^{\text {max }} \geq 0, \forall n \in k_{2}
\end{aligned}
$$

By Fortuny-Amat McCarl linearization [17], equations (26)(31) can be formulated into a mixed-integer linear programming (MILP) problem as follows:

$$
\begin{aligned}
& g_{i} \geq 0, \forall i \in k_{2} \\
& u_{i}^{\text {min }} \geq 0, \forall i \in k_{2} \\
& g_{i} \leq \omega_{i}^{\text {min }} M_{i}^{\text {min }}, \forall i \in k_{2} \\
& u_{i}^{\text {min }} \leq\left(1-\omega_{i}^{\text {min }}\right) M_{i}^{\text {min }}, \forall i \in k_{2} \\
& G_{i}-g_{i} \geq 0, \forall i \in k_{2} \\
& u_{i}^{\text {max }} \geq 0, \forall i \in k_{2} \\
& G_{i}-g_{i} \leq \omega_{i}^{\text {max }} M_{i}^{\text {max }}, \forall i \in k_{2} \\
& u_{i}^{\text {max }} \leq\left(1-\omega_{i}^{\text {max }}\right) M_{i}^{\text {max }}, \forall i \in k_{2} \\
& f_{l}+F_{l} \geq 0, \forall l \in k_{2} \\
& u_{l}^{\text {min }} \geq 0, \forall l \in k_{2} \\
& f_{l}+F_{l} \leq \omega_{l}^{\text {min }} M_{l}^{\text {min }}, \forall l \in k_{2}
\end{aligned}
$$




$$
\begin{aligned}
& u_{l}^{\text {min }} \leq\left(1-\omega_{l}^{\text {min }}\right) M_{l}^{\text {min }}, \forall i \in k_{2} \\
& F_{l}-f_{l} \geq 0, \forall l \in k_{2} \\
& u_{l}^{\text {max }} \geq 0, \forall l \in k_{2} \\
& F_{l}-f_{l} \leq \omega_{l}^{\text {max }} M_{l}^{\text {max }}, \forall l \in k_{2} \\
& u_{l}^{\text {max }} \leq\left(1-\omega_{l}^{\text {max }}\right) M_{l}^{\text {max }}, \forall l \in k_{2} \\
& \delta_{n}+\pi \geq 0, \forall n \in k_{2} \\
& u_{n}^{\text {min }} \geq 0, \forall n \in k_{2} \\
& \delta_{n}+\pi \leq \omega_{n}^{\text {min }} M_{n}^{\text {min }}, \forall n \in k_{2} \\
& u_{n}^{\text {min }} \leq\left(1-\omega_{n}^{\text {min }}\right) M_{n}^{\text {min }}, \forall n \in k_{2} \\
& \pi-\delta_{n} \geq 0, \forall n \in k_{2} \\
& u_{n}^{\text {max }} \geq 0, \forall n \in k_{2} \\
& \pi-\delta_{n} \leq \omega_{n}^{\text {max }} M_{n}^{\text {max }}, \forall n \in k_{2} \\
& u_{n}^{\text {max }} \leq\left(1-\omega_{n}^{\text {max }}\right) M_{n}^{\text {max }}, \forall n \in k_{2} \\
& \omega_{i}^{\text {min }}, \omega_{i}^{\text {max }}, \omega_{l}^{\text {min }}, \omega_{l}^{\text {max }}, \omega_{n}^{\text {min }}, \omega_{n}^{\text {max }} \in\{0,1\}
\end{aligned}
$$

where $M_{i}^{\text {min }}, M_{i}^{\max }, M_{l}^{\text {min }}, M_{l}^{\max }, M_{n}^{\min }, M_{n}^{\max }$ are large enough constants. We now replace constraints (32)(56) into the bilevel program model. This reformulates the optimization problem as a MILP. This MILP model explicitly models the hierarchical dispatch between main-network and sub-network.

\section{Equivalence with Centralized Dispatch}

By checking the KKT conditions of the bilevel optimization model, we are able to prove that our bilevel optimization model (9)-(20) is equivalent to following centralized dispatch model:

$$
\underset{g_{i}, \forall i \in k_{1} \cup k_{2}}{\operatorname{Minimize}} \quad C=\sum_{k} C_{k}
$$

subject to

$$
\begin{aligned}
& \sum_{i \in n} g_{i}-\sum_{j \in n} D_{j}=A^{T} f_{l}, \forall n, l \in k_{1} \cup k_{2} \\
& f_{l}=B_{l} A \delta_{n}, \forall n, l \in k_{1} \cup k_{2} \\
& 0 \leq g_{i} \leq G_{i}, \forall i \in k_{1} \cup k_{2} \\
& 0 \leq f_{l} \leq F_{l}, \forall l \in k_{1} \cup k_{2} \\
& -\pi \leq \delta_{n} \leq \pi, \forall n \in k_{1} \cup k_{2}
\end{aligned}
$$

This mathematical equivalence can be proved further by numerical results in the following section III of this paper. Though model (57)-(62) looks more compact in a mathematical point of view, the practical dispatch complexity in (57)(62) is higher than model (9)-(20). This is mainly due to large volume information of both transmission network and distribution network controlled by one central operator during the centralized dispatch process in (57)-(62).

\section{ILLUSTRATIVE EXAMPLE}

For simplicity, all generation costs are assumed to be linear functions of power production. IEEE 14-bus [18] meshed transmission system as the main network here is connected to IEEE 13-node [18] test feeder sub-network to be the whole test system. Please note IEEE 13-node test feeder is modified here for ease of illustration and we assume the parameter of each branch is equal. The hierarchical dispatch model is implemented in GAMS platform running on windows 7, 64-bit operating system. A personal computer with Intel i7-2760QM $2.4 \mathrm{GHz}$ CPU and 8G RAM is deployed.

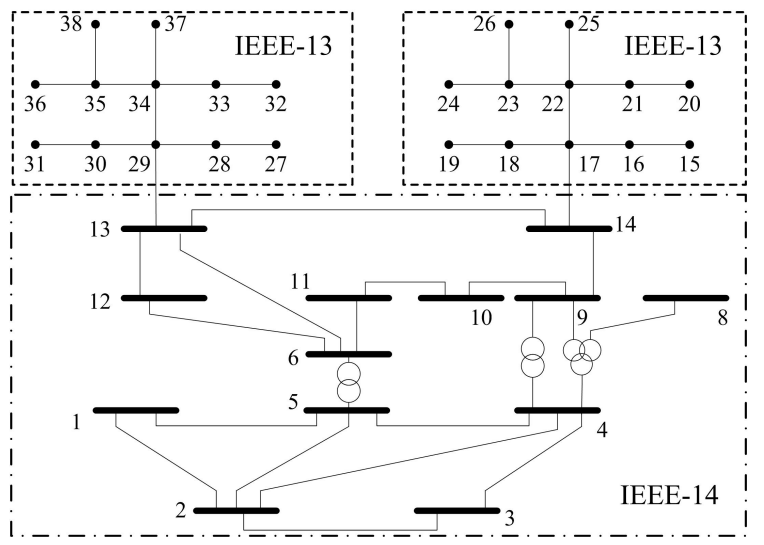

Fig. 2. The IEEE 14-bus meshed transmission system with two IEEE 13-node radial networks connected to bus 13 and bus 14

To simulate DGs successive development stages, three scenarios are explored. We define the first scenario in which variable cost of DGs power production is assumed to be more than conventional generation units in the main network as the pre-smart grid scenario. Second scenario in which variable cost of DGs are equal to conventional generation is defined as smart grid scenario. We even define a third scenario in which power production from DGs cost less than conventional generation as post-smart grid scenario. Data for generation units in different scenarios and demands are listed in the Table I and Table II. In Table I, "Pre-Smart", "Smart" and "Post-Smart" refer to the three considered scenarios correspondingly.

TABLE I

GENERATION DATA

\begin{tabular}{|c|c|c|c|c|c|}
\hline \multirow{2}{*}{ Unit } & \multirow{2}{*}{ Bus } & \multirow{2}{*}{ Capacity (MW) } & \multicolumn{3}{|c|}{ Variable Cost (\$/MWh) } \\
\cline { 4 - 6 } & & & Pre-Smart & Smart & Post-Smart \\
\hline 1 & 1 & 200 & 30 & 30 & 30 \\
\hline 2 & 2 & 100 & 50 & 50 & 50 \\
\hline 3 & 17 & 70 & 60 & 40 & 25 \\
\hline 4 & 25 & 30 & 70 & 25 & 20 \\
\hline 5 & 20 & 10 & 120 & 55 & 35 \\
\hline 6 & 19 & 50 & 90 & 60 & 40 \\
\hline 7 & 32 & 60 & 80 & 35 & 15 \\
\hline 8 & 31 & 40 & 110 & 28 & 18 \\
\hline 9 & 34 & 20 & 100 & 58 & 10 \\
\hline
\end{tabular}

In hierarchical dispatch, bidding functions $(\alpha(\mathbf{x}))$ from sub-networks should be communicated to the main network properly. In fact, knowledge of bidding functions of the subnetwork is of vital importance in hierarchical dispatch. The bidding functions as functions of injected power from the main network in this example for the second scenario are shown in figure 3. The bidding functions (as functions of main network 
TABLE II

DEMAND DATA

\begin{tabular}{|c|c|c|}
\hline Demand & Bus & Capacity (MW) \\
\hline 1 & 6 & 30 \\
\hline 2 & 18 & 20 \\
\hline 3 & 16 & 50 \\
\hline 4 & 23 & 80 \\
\hline 5 & 21 & 60 \\
\hline 6 & 30 & 50 \\
\hline 7 & 27 & 20 \\
\hline 8 & 29 & 50 \\
\hline 9 & 35 & 80 \\
\hline
\end{tabular}

power injection) here are reverse merit-order cost functions of sub-network generation units. When there is no power injection from the main network, all DGs are fully dispatched (assume capacities of DGs are not enough to cover local load and power injection from main network is always required). With the increase of power injection from the main network, power production of DGs are released from the most expensive generation unit to the cheapest generation unit (reverse merit order). Main network operator dispatches the power injection to the sub-network after receiving the bidding from the subnetwork operator. This power injection is actually the networks tie-line power flow. In this way, dispatch task is coordinated by communication between hierarchical network operators.

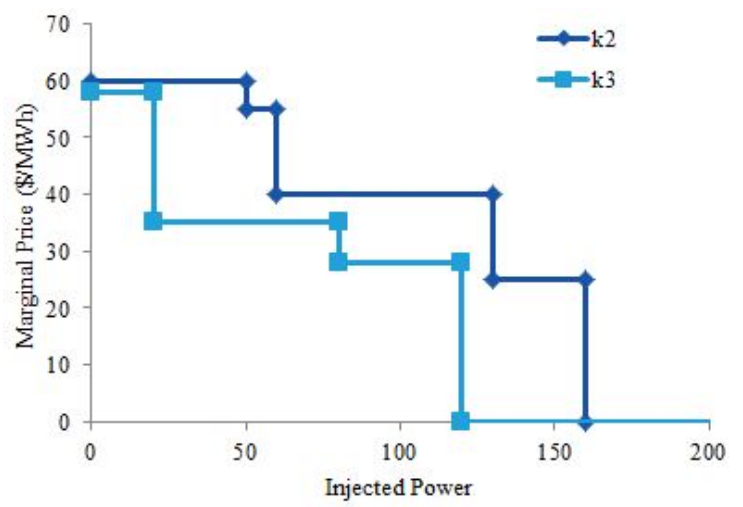

Fig. 3. The Bidding Functions of Sub-networks $\alpha(\mathbf{x})$

\section{A. Dispatch Results}

Two sub-networks are connected with one main network at bus 13 and bus 14 separately. We put two conventional generation units in the main network k1 with large capacity. Seven fully dispatchable DGs are randomly located in the two sub-networks $\mathrm{k} 2$ and $\mathrm{k} 3$. One industrial power demand is located in the main network. The rest eight demand units are located in the two sub-networks. Table III and Figure 4 show the results with traditional centralized dispatch results serving as a benchmark compared with hierarchical dispatch. Power demand remains same in all the scenarios. Centralized dispatch results in Table III are denoted with "T" and hierarchical dispatch results denoting with "H". "G1", "G2", "G3", "G4",
"G5", "G6", "G7", "G8" and "G9" represent power production (MW) from different generation units. As mentioned before, "G1" and "G2" are located in the main network. The rest seven DGs are located in the sub-networks. In Figure 4, "T1", "H1" represent centralized and hierarchical dispatch results in the pre-smart grid scenario. Likewise, "T2", "H2", "T3" and "H3" denote traditional centralized and hierarchical dispatch results for the smart grid scenario and post-grid scenario. The CPLEX solver in GAMS takes around 240 milliseconds to solve the test case for each scenario.

TABLE III

Dispatch RESUlts OF THREE ScEnARIOS

\begin{tabular}{|c|c|c|c|c|c|c|c|}
\hline \multirow{2}{*}{ Network } & \multirow{2}{*}{ Unit } & \multicolumn{2}{|c|}{ Pre-Smart } & \multicolumn{2}{c|}{ Smart } & \multicolumn{2}{c|}{ Post-Smart } \\
\cline { 3 - 8 } & & T & H & T & H & T & H \\
\hline \multirow{2}{*}{ k1 } & G1 & 200 & 200 & 200 & 200 & 200 & 200 \\
& G2 & 100 & 100 & 30 & 30 & 0 & 0 \\
\hline \multirow{4}{*}{ k2 } & G3 & 70 & 70 & 70 & 70 & 70 & 70 \\
& G4 & 30 & 30 & 30 & 30 & 30 & 30 \\
& G5 & 10 & 10 & 10 & 10 & 10 & 10 \\
& G6 & 0 & 0 & 0 & 0 & 10 & 10 \\
\hline \multirow{3}{*}{ k3 } & G7 & 30 & 30 & 60 & 60 & 60 & 60 \\
& G8 & 0 & 0 & 40 & 40 & 40 & 40 \\
& G9 & 0 & 0 & 0 & 0 & 20 & 20 \\
\hline \multicolumn{2}{|c|}{ Cost (\$) } & 20900 & 20900 & 14820 & 14820 & 10920 & 10920 \\
\hline
\end{tabular}

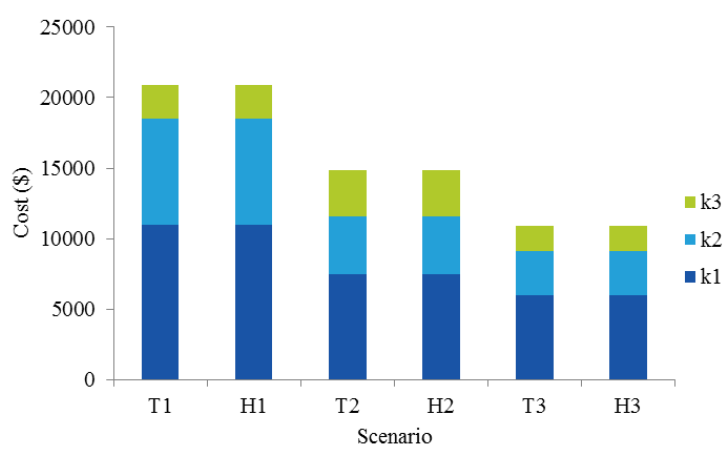

Fig. 4. Cost in Three Scenarios

Hierarchical dispatch achieves same results as traditional centralized dispatch (global optimization) in all the three considered scenarios. This is expected by the equivalence argument in part D of section II in this paper. In all scenarios, generation in the main network are always dispatched for backbone base load support. This is because of small capacity assumptions for DGs. In the pre-smart grid scenario, generation units located in the main network are always prior to be dispatched. This makes sense when energy offered by DGs cost more than conventional bulk generation. If $\mathrm{CO}_{2}$ taxes and other benefits of DGs such as improved voltage profile in distribution system are considered in the future, model parameters will be modified and bulk generation in the main network will not maintain dispatch priority any more i.e. DGs are competitive with bulk generation. This is illustrated by the smart grid scenario. The post-smart grid scenario is rather bold assumption. In this future scenario, generation cost 
dramatically drop down and the demand is served with lowest cost. This third scenario shows that when DGs variable cost are less than bulk generation hierarchical dispatch cost keeps same with centralized dispatch. So hierarchical dispatch achieves same dispatch results at the advantage of reducing dispatch complexity.

\section{B. Distribution Network Nodal Pricing}

After aforementioned MILP model is solved by CPLEX solver in GAMS, the solutions of binary variables are obtained. By fixing binary variables to be the optimum solutions, the MILP problem is then transformed to a linear programming problem (LP). The solutions of associated dual variables $\lambda_{n}^{p}$ in this LP are nodal prices. Nodal prices in the smart grid scenario are demonstrated in figure 5. As hierarchical dispatch achieves exactly same results with centralized dispatch, the prices also coincide. If there are congestions, nodal prices

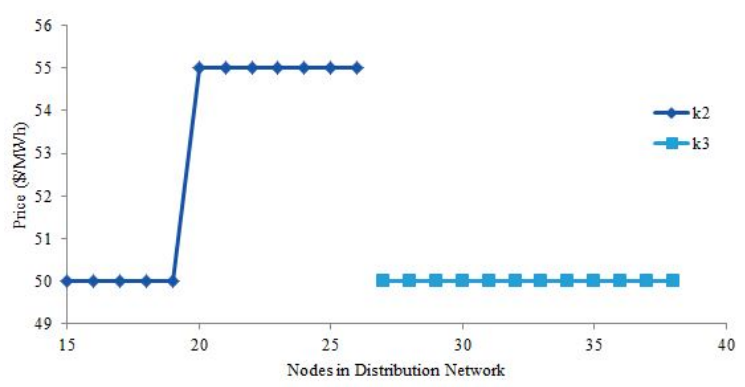

Fig. 5. Distribution Networks Price

in distribution system vary among different nodes. In this case, one congested branch in sub-network $\mathrm{k} 2$ divides the distribution network into two different price areas. Local DGs become the marginal generators for this sub-network. This high price signal during congestion period gives clear incentives for local DGs investment and network investment. For sub-network k3, there are no congested branches. So sub-network k3 demonstrates uniform prices. Nodal pricing in distribution network offers locational economic signal to direct distribution system operation, investment and demand response. This benefit can not be obtained from traditional regional pricing as no variation with locations inside the region exists.

\section{CONCLUSion}

To coordinate economic dispatch in the context of hierarchical network operators and facilitate distribution network pricing, a hierarchical dispatch structure is proposed and explored. Features and advantages of hierarchical dispatch are explained. By checking KKT conditions of the proposed hierarchical dispatch model, we are able to prove that it is equivalent with centralized dispatch model. So global optimization can be achieved by the hierarchical dispatch. This argument is further proved by numerical results. Scenarios representing successive DGs development stages are analyzed. Numerical results demonstrate feasibility of hierarchical dispatch. Hierarchical dispatch achieves same results to centralized dispatch in all three scenarios as expected. Traditional centralized dispatch for both transmission and distribution system is complex and requires a large volume of information. This is impractical to implement for efficient distribution pricing if DGs penetration are increasing in future smart grid. Hierarchical dispatch decomposes the dispatch task into sub tasks carried out by individual regional transmission and distribution operators. In this way, communication and computation burdens are fairly relaxed. Distribution network nodal prices obtained by hierarchical dispatch are calculated and reported. The efficiency of distribution network nodal prices need to be further investigated.

\section{REFERENCES}

[1] J. Reneses and M. Rodriguez Ortega, "Distribution pricing: theoretical principles and practical approaches," Generation, Transmission Distribution, IET, vol. 8, no. 10, pp. 1645-1655, October 2014.

[2] D. R. Biggar and M. R. Hesamzadeh, The Economics of Electricity Markets. IEEE Press \& Wiley, 2014.

[3] P. M. Sotkiewicz and J. M. Vignolo, "Nodal pricing for distribution networks: efficient pricing for efficiency enhancing dg," Power Systems, IEEE Transactions on, vol. 21, no. 2, pp. 1013-1014, 2006.

[4] R. Li, Q. Wu, and S. Oren, "Distribution locational marginal pricing for optimal electric vehicle charging management," Power Systems, IEEE Transactions on, vol. 29, no. 1, pp. 203-211, 2014.

[5] S. Widergren, C. Marinovici, T. Berliner, and A. Graves, "Real-time pricing demand response in operations," in Power and Energy Society General Meeting, 2012 IEEE, 2012, pp. 1-5.

[6] F. Sahriatzadeh, P. Nirbhavane, and A. Srivastava, "Locational marginal price for distribution system considering demand response," in North American Power Symposium (NAPS), 2012, 2012, pp. 1-5.

[7] N. D. of public Service, "Reforming the energy vision," in Proceeding on Motion of Commission in Regard to Reforming the Energy Vision, 2014.

[8] S. M. Farrokh Rahimi. (2014, June) From iso to dso [Online]. Available: http://mag.fortnightly.com/article/From+ISO+to+ DSO/1727340/212098/article.html

[9] F. Li, "Recent developments in common distribution network pricing in Great Britain," in Energy Market (EEM), 2010 7th International Conference on the European, 2010, pp. 1-5.

[10] R. Mudumbai and S. Dasgupta, "Distributed control for the smart grid: The case of economic dispatch," in Information Theory and Applications Workshop (ITA), 2014, 2014, pp. 1-6.

[11] H. Karami, M. Sanjari, S. Hosseinian, and G. Gharehpetian, "An optimal dispatch algorithm for managing residential distributed energy resources," IEEE Transactions on Smart Grid, vol. 5, no. 5, pp. 23602367, 2014

[12] A. Fazeli, M. Sumner, C. Johnson, and E. Christopher, "Coordinated optimal dispatch of distributed energy resources within a smart energy community cell," in Innovative Smart Grid Technologies (ISGT Europe), 2012 3rd IEEE PES International Conference and Exhibition on, 2012, pp. $1-10$.

[13] A. Conejo and J. Aguado, "Multi-area coordinated decentralized DC optimal power flow," vol. 13, no. 4, pp. 1272-1278, 1998.

[14] Y. Zhang and F. Li, "Network pricing for high voltage radial distribution networks," in Power and Energy Society General Meeting, 2011 IEEE, 2011, pp. 1-5.

[15] V. Loia and A. Vaccaro, "Decentralized economic dispatch in smart grids by self-organizing dynamic agents," IEEE Transactions on Systems, Man, and Cybernetics: Systems, vol. 44, no. 4, pp. 397-408, 2014.

[16] F. Meng and B. Chowdhury, "Distribution lmp-based economic operation for future smart grid," in Power and Energy Conference at Illinois (PECI), 2011 IEEE, 2011, pp. 1-5.

[17] S. Gabriel, A. Conejo, J. Fuller, B. Hobbs, and C. Ruiz, Complementarity Modelling in Energy Markets. Springer, 2012.

[18] The university of washington power systems test case archive. [Online]. Available: http://www.ee.washington.edu/research/pstca/ 